Research Paper

\title{
Effect of metabolic syndrome components on the risk of malignancy in patients with gallbladder lesions
}

\author{
Zheng Deng, ${ }^{1}$ Yan Xuan², Xinxing Li³,4, William J Crawford5, Zhiqing Yuan', Zhoukan Chen ${ }^{6}$, Anastasia \\ Brooks 7,8 , Yanyan Song ${ }^{9}$, Haolu Wang7,8, Xiaowen Liang7,8, Tao Chen ${ }^{1,10^{\bowtie}}$ \\ 1. Department of General Surgery, South Campus, Renji Hospital, School of Medicine, Shanghai Jiaotong University, Shanghai, 201112, China \\ 2. Department of Endocrinology, Luwan Branch, Ruijin Hospital, School of Medicine, Shanghai Jiaotong University, Shanghai, 200020, China \\ 3. Department of Gastroenterological Surgery, Tongji Hospital, School of Medicine, Tongji University, Shanghai, 200065, China \\ 4. Department of General Surgery, Changzheng Hospital, The Second Military Medical University, Shanghai, 200003, China \\ 5. Department of Medical Sciences, University of Oxford, Oxford OX3 9DU, United Kingdom \\ 6. Department of General Surgery, Luwan Branch, Ruijin Hospital, School of Medicine, Shanghai Jiaotong University, Shanghai, 200020, China \\ 7. The University of Queensland Diamantina Institute, The University of Queensland, Brisbane, QLD, 4102, Australia \\ 8. Gallipoli Medical Research Institute, Greenslopes Private Hospital, Brisbane, QLD, 4120, Australia \\ 9. Department of biostatistics, clinical research institute, School of Medine, Shanghai Jiaotong University, Shanghai, 200025, China \\ 10. Department of Biliary-Pancreatic Surgery, Renji Hospital, School of Medicine, Shanghai Jiao Tong University, Shanghai, 200127, China \\ Zheng Deng, Yan Xuan and Xinxing Li contributed equally to this work. \\ $\triangle$ Corresponding author: Tao Chen, Tel: +86 215388 2125, Email: dr_chentao78@163.com \\ (C) The author(s). This is an open access article distributed under the terms of the Creative Commons Attribution License (https://creativecommons.org/licenses/by/4.0/). \\ See http://ivyspring.com/terms for full terms and conditions.
}

Received: 2020.10.17; Accepted: 2020.12.21; Published: 2021.01.01

\begin{abstract}
Background: Gallbladder lesions have become more common nowadays. But there is limited evidence-based guidance on surveillance of these patients for malignancy. Predicting malignancy could help clinicians better manage this condition and improve the prognosis. We evaluated the independent and joint effects of metabolic syndrome components on the risk of malignancy among patients with gallbladder lesions.

Methods: Using a multicenter database, consecutive patients with pathologically confirmed gallbladder lesions between 2012 and 2019 were identified. Univariate and multivariate logistic regression analyses were used to evaluate the effects of metabolic syndrome components (diabetes, hypertension, dyslipidemia and obesity) as additive or combined indicators for the risk of malignancy. Unadjusted and adjusted odds ratios were calculated.

Results: Of the 625 patients, 567 patients were identified with benign gallbladder lesions and 58 patients with gallbladder cancer (GBC). GBC group had less obesity but more dyslipidemia. Among all metabolic syndrome components, only dyslipidemia was significantly associated with GBC (odds ratio $2.674,95 \%$ confidence interval 1.173-6.094). Dyslipidemia was an independent risk factor for malignancy (adjusted odds ratio $2.164,95 \%$ confidence interval 1.165-4.021), regardless of whether the other risk factors and metabolic syndrome components were combined. Patients with decreased high-density lipoprotein had 3.035-fold higher risk of malignancy (adjusted odds ratio 3.035, 95\% confidence interval 1.645-5.600).

Conclusions: Dyslipidemia is associated with a 2.674-fold increase in the risk of malignancy in patients with gallbladder lesions. Dyslipidemia is an independent risk factor for malignancy, regardless of the presence of the other risk factors and metabolic syndrome components.
\end{abstract}

Key words: Gallbladder lesions, Benign gallbladder polyps, Gallbladder cancer, Metabolic syndrome components, Dyslipidemia, High density lipoprotein

\section{Introduction}

Gallbladder lesions have become more common nowadays with the increased use of ultrasound [1]. The incidence rate of gallbladder lesions is approximately $5-9.9 \%$ of the population [2]. Cholesterol gallbladder polyps are the most common type of benign gallbladder polyps (BGP). Some BGP, 
such as adenomas, are considered with malignant potential [1]. About 3-8\% of all gallbladder lesions are malignant [3]. Current European consensus guideline suggests that all gallbladder lesions greater than 10 $\mathrm{mm}$ should be surgically removed, and those between 6 and $9 \mathrm{~mm}$ with co-existing presence of high risk factors are deemed to justify cholecystectomy [4]. However, there is limited evidence-based guidance on how to manage patients who were not offered surgical treatment [5]. It has been suggested patients with gallbladder lesions less than $10 \mathrm{~mm}$ could be followed conservatively $[1,4]$. For such surveillance to be cost-effective, better risk stratification is needed to guide targeted surveillance. Predicting malignancy by better understanding risk factors would also allow clinicians to more effectively plan secondary prevention efforts of gallbladder cancer (GBC) for patients with gallbladder lesions [6, 7].

The metabolic syndrome components (diabetes, hypertension, dyslipidemia and obesity) are readily identifiable and potentially modifiable, rendering them as ideal targets not only for risk stratification but also for risk modification, which would be useful to both predicting prognosis and preventing complications. Some manifestations of metabolic syndrome (such as diabetes, hyperlipidemia and obesity) are the risk factors for gallbladder stones or lesions [8]. Diabetes was associated with biliary tract cancers [9].

However, until now, it is still controversial whether the metabolic syndrome components could be involved in the malignant risk scoring systems for gallbladder lesions. Available studies followed only a limited number of patients with incomplete risk factor data [10, 11]. There remains doubt about the association between metabolic syndrome components and the risk of biliary tract cancers [12]. The frequent interaction and co-occurrence of these metabolic syndrome components also complicates the analysis of each component's specific contribution to the risk of malignancy in patients with gallbladder lesions.

To fill this gap, we conducted a retrospective cohort study of 625 patients with pathologically confirmed gallbladder lesions from three hospitals in China. The independent and joint effects of syndrome components on the risk of malignancy were evaluated.

\section{Method}

\section{Study population and laboratory tests}

Using a multicenter database, a total of 625 consecutive postoperative patients who pathologically diagnosed with gallbladder lesions from February 2012 and December 2019 at three
Chinese hospitals (Renji Hospital, Ruijin Hospital and Shanghai Changzheng Hospital) were identified. A standardised data form was created to collect all relevant information including age, gender, body mass index, hypertension, diabetes, the data of ultrasonography including number of gallbladder lesions (single or multiple), accompanying with stones and maximum diameter of lesions and laboratory findings including fasting blood glucose, liver function tests and lipid profiles. The inclusion criteria for the patients with gallbladder lesions were as follows: (a) patients underwent surgical treatment and had pathologically confirmed diagnosis; (b) examination with ultrasonography was performed within 1 month before surgery. We excluded patients diagnosed with other cancers within the five years before the diagnosis of gallbladder lesions to ensure metastatic cancers were excluded. Patients younger than 18 years or with missing any of the required data were excluded. Dyslipidemia was defined when any of the following criteria were satisfied: 1) hypercholesterolemia: $\geq 6.2 \mathrm{mmol} / \mathrm{L} ; 2$ ) hypertriglyceridemia: $\geq 2.3 \mathrm{mmol} / \mathrm{L}(200 \mathrm{mg} / \mathrm{dL})$; 3) low high density lipoprotein (HDL) cholesterol: $<1.03$ $\mathrm{mmol} / \mathrm{L}(40 \mathrm{mg} / \mathrm{dL}$ ) in men and $<1.29 \mathrm{mmol} / \mathrm{L}$ (50 $\mathrm{mg} / \mathrm{dL}$ ) in women. Obese was identified by BMI $\geq$ $25 \mathrm{~kg} \mathrm{~m}^{-2}$ according to the WHO classification for Asian populations. The study was conducted in accordance with the Declaration of Helsinki and was approved by the Institutional Ethical Review Committee of Renji Hospital (Ethical Approval Number 2016-045).

\section{Histopathology}

All gallbladder lesions underwent an independent review by two pathologists. In this study, BGP include cholesterol polyps, inflammatory polyps, adenoma, adenomyomatosis, cholesterolosis and hyperplasia. GBC only include adenocarcinoma [13].

\section{Statistical analysis}

Data on continuous variables were expressed as mean \pm standard deviation (SD), and the categorical variables are summarised as frequencies and percentages. $T$ tests were conducted for continuous variables and $\chi 2$ tests for categorical variables. Odds ratios (OR), 95\% confidence interval (CI) and $P$ values were calculated for each variable. The multivariate logistic regression models were constructed to examine the risk of malignancy. The univariate and multivariate models were constructed to test the joint association between dyslipidemia and the other metabolic syndrome components and restricted one risk factor without the others. Models were adjusted 
for age and gender. The results were considered statistically significant when $P$ values were $<0.05$. The adjusted ORs and 95\% CIs were calculated for each parameter estimate. All statistical analyses were performed by SPSS version 25 (IBM Co., Armonk, NY, USA).

\section{Results}

\section{Patient characteristics}

We identified 625 patients with gallbladder lesions in total. As shown in Table 1, 567 patients had BGP and 58 had GBC. There was no significant difference in gender between the two groups. The mean age of the two groups was $49.94 \pm 12.73$ and $65.84 \pm 9.98$ years old, respectively. Patients in GBC group were significantly older than those in BGP group $(P<0.001)$ or the gallbladder adenomatous polyp group $(P<0.001$, Supplementary Table 1$)$. Multiple lesions were found more frequently in patients with BGP than in those with GBC $(64.6 \%$ vs $29.3 \%, P<0.001)$. Comparing to the BGP group, the level of total bilirubin was significantly higher in GBC group $(P=0.001$, Table 1$)$ or adenomatous polyp group $(P=0.009$, Supplementary Table 1$)$. Among all patients, the BGP, adenomatous polyp and GBC had different mean lesion diameters of $6.49 \pm 4.60 \mathrm{~mm}$, $9.27 \pm 5.70 \mathrm{~mm}$ and $26.26 \pm 22.66 \mathrm{~mm}$, respectively $(P$ $<$ 0.001). Accompanying gallstones was more common in the GBC group (3.4\%) than the BGP group (20.8\%, $P=0.001)$ or the adenomatous polyp group $(25.4 \%, P=0.001$, Supplementary Table 1$)$.

Table 1. Baseline characteristics of patients with gallbladder lesions

\begin{tabular}{llll}
\hline & $\begin{array}{l}\text { Benign gallbladder } \\
\text { polyps }(\mathrm{n}=567)\end{array}$ & $\begin{array}{l}\text { Gallbladder } \\
\text { cancer }(\mathrm{n}=58)\end{array}$ & Pvalue \\
\hline Gender & $284(50.1)$ & $29(50.0)$ & 0.990 \\
Male & $283(49.9)$ & $29(50.0)$ & \\
Female & $49.94(12.73)$ & $65.84(9.98)$ & $<\mathbf{0 . 0 0 1}$ \\
Age, mean (SD) & & & $<\mathbf{0 . 0 0 1}$ \\
Number of polyps & $201(35.4)$ & $41(70.7)$ & \\
Single & $366(64.6)$ & $17(29.3)$ & \\
Multiple & $18.40(47.80)$ & $44.15(88.99)$ & $\mathbf{0 . 0 0 1}$ \\
Total bilirubin & $6.49(4.60)$ & $26.26(22.66)$ & $<\mathbf{0 . 0 0 1}$ \\
Maximum diameter of & & & \\
polyps & $118(20.8)$ & $2(3.4)$ & $\mathbf{0 . 0 0 1}$ \\
With stones & $202(35.6)$ & $13(22.4)$ & $\mathbf{0 . 0 4 4}$ \\
BMI $\geq 25$ & $275(48.5)$ & $34(58.6)$ & 0.142 \\
Hypertension & $84(11.3)$ & $10(17.2)$ & 0.181 \\
Diabetes & $239(42.2)$ & $37(63.8)$ & $\mathbf{0 . 0 0 2}$ \\
Dyslipidemia & $87(15.3)$ & $6(10.3)$ & 0.308 \\
Total cholesterol $\geq 6.2$ & $72(12.7)$ & $9(15.5)$ & 0.543 \\
TG $\geq 2.3$ & $144(25.4)$ & $31(53.4)$ & $<\mathbf{0 . 0 0 1}$ \\
Decreased HDL &
\end{tabular}

TG: Triglycerides; HDL, high-density lipoprotein. BMI: body mass index.

Obesity was more common in BGP group compared to GBC group (Table $1,35.6 \%$ vs $22.4 \%, P=$ 0.044). Dyslipidemia was more common in GBC group than BGP group (63.8\% vs $42.2 \%, \mathrm{P}=0.002)$. Decreased HDL was more common in the GBC group than BGP or adenomatous polyp groups $(P<0.001$, Supplementary Table 1). There was no significant difference in total cholesterol $(15.3 \%$ vs $10.3 \%)$, triglycerides (TG, $12.7 \%$ vs $15.5 \%$ ), hypertension ( $48.5 \%$ vs 58.6$)$ and diabetes ( $11.3 \%$ vs $17.2 \%)$ between the BGP and GBC groups.

\section{Independent risk factors for GBC}

Univariate and multivariable analyses were performed to identify independent risk factors for GBC (Table 2). A single factor regression analysis on all indicators revealed that age, number of polyps, total bilirubin, maximum diameter of polyps, accompanying stones, BMI and dyslipidemia were associated with an increased risk of GBC. Dyslipidemia was associated with a 2.4-fold increase in the odds of GBC $(P=0.002)$.

Table 2. Univariate and multivariate analyses of the risk factors for gallbladder lesions

\begin{tabular}{|c|c|c|c|c|c|c|}
\hline & \multicolumn{3}{|c|}{ Univariate analysis } & \multicolumn{3}{|c|}{ Multivariate analysis } \\
\hline & OR & $95 \% \mathrm{CI}$ & $\mathrm{P}$ & $\begin{array}{l}\text { Adjusted } \\
\text { OR }\end{array}$ & $95 \% \mathrm{CI}$ & $\mathrm{P}$ \\
\hline Age & 1.130 & $1.095-1.165$ & $<0.001$ & & & \\
\hline Gender & 0.996 & $0.580-1.711$ & 0.990 & & & \\
\hline Number of polyps & 0.228 & $0.126-0.411$ & $<0.001$ & 1.307 & $0.566-3.020$ & 0.531 \\
\hline Total bilirubin & 1.005 & $1.001-1.008$ & 0.005 & 1.003 & $0.998-1.009$ & 0.222 \\
\hline $\begin{array}{l}\text { Maximum } \\
\text { diameter of polyps }\end{array}$ & 1.180 & $1.133-1.230$ & $<0.001$ & 1.162 & $1.107-1.219$ & $\begin{array}{l}< \\
0.001\end{array}$ \\
\hline Stones & 0.136 & $0.033-0.565$ & 0.006 & 0.178 & $0.036-0.882$ & 0.034 \\
\hline Hypertension & 1.504 & $0.870-2.602$ & 0.144 & 1.116 & $0.478-2.609$ & 0.799 \\
\hline Diabetes & 1.637 & $0.790-3.395$ & 0.185 & 0.768 & $0.240-2.465$ & 0.768 \\
\hline Dyslipidemia & 2.418 & $1.380-4.237$ & 0.002 & 2.674 & $1.173-6.094$ & 0.019 \\
\hline $\mathrm{BMI} \geq 25$ & 0.522 & $0.275-0.991$ & 0.047 & 0.552 & $0.228-1.338$ & 0.188 \\
\hline
\end{tabular}

Model adjusted for age and sex.

$\mathrm{OR}$, odds ratio; $\mathrm{CI}$, confidence interval;

These risk factors were then included in the multivariate analysis, which was adjusted for age and gender. As shown in Table 2, dyslipidemia and size of polyps were associated with a 2.6-fold and 1.162-fold increase in risk of malignancy in patients with gallbladder lesions. For patients with neoplastic gallbladder lesions, decreased HDL was associated with a 5-fold increase in risk of malignancy in $(95 \%$ CI: 1.502-16.801, $P=0.009$, Supplementary Table 2).

\section{Joint associations of metabolic syndrome components with GBC}

Metabolic syndrome components often coexist in patients. Then we examined whether a specific combination of them was associated with an increased risk of GBC. Table 3 summarised the different types of metabolic characteristics in the two groups. Six combinations of metabolic conditions were more common in GBC group than BGP group, including diabetes without $\mathrm{BMI} \geq 25$, dyslipidemia without $\mathrm{BMI}$ 
$\geq 25$, hypertension without $\mathrm{BMI} \geq 25$, diabetes without hypertension, dyslipidemia and hypertension, and dyslipidemia and diabetes.

Table 3. Types of metabolic traits of patients with gallbladder lesions

\begin{tabular}{llll}
\hline Metabolic syndrome components & $\begin{array}{l}\text { Benign gallbladder } \\
\text { polyps (567) }\end{array}$ & $\begin{array}{l}\text { Gallbladder } \\
\text { cancer (58) }\end{array}$ & Pvalue \\
\hline Hypertension & $275(48.5)$ & $34(58.6)$ & 0.142 \\
Hypertension Excluding Diabetes & $230(40.6)$ & $29(50.0)$ & 0.165 \\
Hypertension Excluding & $152(26.8)$ & $10(17.2)$ & 0.113 \\
Dyslipidemia & & & \\
Hypertension Excluding BMI $\geq 25$ & $153(27.0)$ & $24(41.1)$ & $\mathbf{0 . 0 2 0}$ \\
Diabetes & $84(11.3)$ & $10(17.2)$ & 0.181 \\
Diabetes Excluding Hypertension & $19(3.4)$ & $5(8.6)$ & $\mathbf{0 . 0 4 7}$ \\
Diabetes Excluding BMI $\geq 25$ & $35(6.2)$ & $7(12.1)$ & 0.088 \\
Diabetes Excluding Dyslipidemia & $38(6.7)$ & $2(3.4)$ & 0.335 \\
Dyslipidemia & $239(42.2)$ & $37(63.8)$ & $\mathbf{0 . 0 0 2}$ \\
Dyslipidemia Excluding & $116(20.5)$ & $13(22.4)$ & 0.726 \\
Hypertension & & & \\
Dyslipidemia Excluding Diabetes & $213(37.6)$ & $29(50.0)$ & 0.064 \\
Dyslipidemia Excluding BMI $\geq 25$ & $147(25.9)$ & $29(50.0)$ & $<0.001$ \\
Dyslipidemia and Hypertension & $123(21.7)$ & $24(41.4)$ & $\mathbf{0 . 0 0 1}$ \\
Dyslipidemia and Diabetes & $26(4.6)$ & $8(13.8)$ & $\mathbf{0 . 0 0 3}$ \\
Dyslipidemia and BMI $\geq 25$ & $92(16.2)$ & $8(13.8)$ & 0.630 \\
BMI $\geq 25$ & $202(35.6)$ & $13(22.4)$ & $\mathbf{0 . 0 4 4}$ \\
\hline
\end{tabular}

To verify whether these factors increased the risk of malignancy, both univariate and multivariate analyses were performed (Table 4). In the univariate analysis, we found that hypertension without BMI $\geq$ 25 (OR=1.910, 95\% CI: 1.097-3.325), with dyslipidemia without $\mathrm{BMI} \geq 25$ (OR=2.857, 95\% CI: 1.652-4.924), with both dyslipidemia and hypertension $(\mathrm{OR}=2.548$, 95\% CI: 1.456-4.458) and with both dyslipidemia and diabetes (OR=3.329, 95\% CI: 1.432-7.740) were associated with risk of malignancy in patients with gallbladder lesions. The multivariate analysis model was adjusted for age and gender, and dyslipidemia excluding diabetes group and dyslipidemia excluding obese group were significantly associated with GBC. Dyslipidemia without diabetes, and dyslipidemia without $\mathrm{BMI} \geq 25$ were both associated with an approximately 1.9 - and 2.5 -fold increase in the risk of malignancy in patients with gallbladder lesions. The trait of hypertension without dyslipidemia seem to be a protective factor for patients $(\mathrm{OR}=0.438,95 \% \mathrm{CI}$ : 0.201-0.953). There was no other significant interaction among dyslipidemia and other risk factors. These results indicated that dyslipidemia is an independent risk factor for increase in the risk of malignancy, regardless of the presence of other metabolic syndrome components.

\section{Analysis of dyslipidemia types for GBC}

Our results show that dyslipidemia is a risk factor for GBC. We then examined this association with different dyslipidemia types, including hypercholesterolemia, TG, increased low density lipoprotein (LDL) and decreased high density lipoproteins (HDL). In both univariate and multivariable analyses, dyslipidemia and decreased HDL were closely associated with increased the risk of malignancy in patients with gallbladder lesions (Table 5) or with neoplastic gallbladder lesions (Supplementary Table 2), while elevated total cholesterol level or TG level had no significantly impact on the risk of malignancy.

Table 4. Univariate and multivariate analyses of the association of types of metabolic syndrome components for gallbladder lesions

\begin{tabular}{|c|c|c|c|c|c|c|}
\hline & \multicolumn{3}{|c|}{ Univariate analysis } & \multicolumn{3}{|c|}{ Multivariate analysis } \\
\hline & OR & $95 \% \mathrm{CI}$ & $\mathrm{P}$ & $\begin{array}{l}\text { Adjusted } \\
\text { OR }\end{array}$ & $95 \% \mathrm{CI}$ & $\mathrm{P}$ \\
\hline Age & 1.130 & $1.095-1.165$ & $\begin{array}{l}< \\
0.001\end{array}$ & & & \\
\hline Gender & 0.996 & $0.580-1.711$ & 0.990 & & & \\
\hline Hypertension & 1.504 & $0.870-2.602$ & 0.144 & 0.911 & $0.496-1.675$ & 0.765 \\
\hline $\begin{array}{l}\text { Hypertension Excluding } \\
\text { Diabetes }\end{array}$ & 1.465 & $0.853-2.518$ & 0.167 & 1.221 & $0.671-2.222$ & 0.512 \\
\hline $\begin{array}{l}\text { Hypertension Excluding } \\
\text { Dyslipidemia }\end{array}$ & 0.569 & $0.281-1.153$ & 0.117 & 0.438 & $0.201-0.953$ & 0.038 \\
\hline $\begin{array}{l}\text { Hypertension Excluding } \\
\text { BMI } \geq 25\end{array}$ & 1.910 & $1.097-3.325$ & 0.022 & 1.227 & $0.662-2.273$ & 0.516 \\
\hline Diabetes & 1.637 & $0.790-3.395$ & 0.185 & 0.754 & $0.326-1.741$ & 0.508 \\
\hline $\begin{array}{l}\text { Diabetes Excluding } \\
\text { Hypertension }\end{array}$ & 2.721 & $0.977-2.721$ & 0.056 & 1.821 & $0.559-5.929$ & 0.320 \\
\hline $\begin{array}{l}\text { Diabetes Excluding BMI } \geq \\
25\end{array}$ & 2.086 & $0.882-4.934$ & 0.094 & 0.962 & $0.353-2.619$ & 0.962 \\
\hline $\begin{array}{l}\text { Diabetes Excluding } \\
\text { Dyslipidemia }\end{array}$ & 0.497 & $0.117-2.116$ & 0.344 & & & \\
\hline Dyslipidemia & 2.418 & $1.380-4.237$ & 0.002 & 2.164 & $1.165-4.021$ & 0.015 \\
\hline $\begin{array}{l}\text { Dyslipidemia Excluding } \\
\text { Hypertension }\end{array}$ & 1.128 & $0.586-2.151$ & 0.726 & & & \\
\hline $\begin{array}{l}\text { Dyslipidemia Excluding } \\
\text { Diabetes }\end{array}$ & 1.162 & $0.967-2.858$ & 0.066 & 1.915 & $1.039-3.526$ & 0.037 \\
\hline $\begin{array}{l}\text { Dyslipidemia Excluding } \\
\text { BMI } \geq 25\end{array}$ & 2.857 & $1.652-4.924$ & $\begin{array}{l}< \\
0.001\end{array}$ & 2.525 & $1.365-4.669$ & 0.003 \\
\hline $\begin{array}{l}\text { Dyslipidemia and } \\
\text { Hypertension }\end{array}$ & 2.548 & $1.456-4.458$ & 0.001 & 1.721 & 0.927-3.195 & 0.085 \\
\hline Dyslipidemia and Diabetes & 3.329 & $1.432-7.740$ & 0.005 & 1.406 & $0.544-3.635$ & 0.482 \\
\hline Dyslipidemia and BMI $\geq 25$ & 0.826 & $0.379-1.800$ & 0.631 & & & \\
\hline $\mathrm{BMI} \geq 25$ & 0.522 & $0.275-0.991$ & 0.047 & 0.528 & $0.262-1.067$ & 0.075 \\
\hline
\end{tabular}

Model adjusted for age and sex.

Table 5. Comparison of different indexes of dyslipidemias for gallbladder lesions

\begin{tabular}{|c|c|c|c|c|c|c|}
\hline & \multicolumn{3}{|c|}{ Univariate analysis } & \multicolumn{3}{|c|}{ Multivariate analysis } \\
\hline & OR & $95 \% \mathrm{CI}$ & $\mathrm{P}$ & $\begin{array}{l}\text { Adjusted } \\
\text { OR }\end{array}$ & $95 \% \mathrm{CI}$ & $P$ \\
\hline Dyslipidemia & 2.418 & $1.380-4.237$ & 0.002 & 2.164 & $1.165-4.021$ & 0.015 \\
\hline $\begin{array}{l}\text { Total cholesterol } \\
\geq 6.2\end{array}$ & 0.637 & $0.265-1.528$ & 0.312 & & & \\
\hline $\mathrm{TG} \geq 2.3$ & 1.263 & $0.595-2.680$ & 0.543 & & & \\
\hline Decreased HDL & 3.373 & $1.947-5.843$ & $\begin{array}{l}< \\
0.001\end{array}$ & 3.035 & $1.645-5.600$ & $\begin{array}{l}< \\
0.001\end{array}$ \\
\hline
\end{tabular}

Model adjusted for age and sex.

\section{Discussion}

Although the incidence of GBC is low, its prognosis is extremely poor $[14,15]$ with a 5 -year survival rate less than $20 \%$ [16]. Predicting the risk of malignancy of gallbladder lesions could help clinicians manage this condition and potentially improve the prognosis [17]. A recent systematic 
review revealed the role of diabetes in gallbladder diseases [18]. However, the specific individual diabetic or other metabolic risk factors are still unclear. This is the first study that elucidated the association between detailed metabolic syndrome components (diabetes, hypertension, obese and dyslipidemia) and the risk of malignancy in patients with gallbladder lesions.

We found that the GBC group had older age, larger size of polyps, more common presence of gallbladder stones, obesity and dyslipidemia compared with the BGP group. In the multivariate analysis, single polyp and elevated bilirubin level had no significant association with malignancy, but dyslipidemia was a significant risk factor for GBC. The older age and larger polyp diameter have been reported as risk factors of GBC $[10,19,20]$, which were similar to our results.

In this study, dyslipidemia was identified as an independent risk factor for malignancy, regardless of the presence of diabetes, hypertension and obese. Dyslipidemia was present in $44 \%$ of patients with gallbladder lesions and associated with a 2.6-fold increased risk of malignancy. These findings suggest that dyslipidemia may contribute to the malignancy progression of gallbladder lesions. Elevated total cholesterol, elevated TG and decreased HDL have been associated with an $18 \%, 15 \%$, and $20 \%$ increased risk of cancer, respectively [21]. Previous studies also indicated that statin, a cholesterol-lowering medication, could decrease the risk of developing cancers [22]. Dyslipidemia also closely related to oxidative stress. Dyslipidemia downregulated the HDL antioxidant/ anti-inflammatory function and increased oxidative stress and inflammatory, which have been proven to be contributing factors for cancers [23].

Individual metabolic syndrome components are closely related and frequently co-occur. Dyslipidemia is usually a feature in patients with type 2 diabetes and obesity. Patients with obesity and diabetes frequently exhibit elevated circulating cholesterol, TG, and reduced HDL [24]. Diabetes, obesity and dyslipidemia contribute to the development and progression of many malignancies [25]. Thus, we further evaluated the effects of metabolic syndrome components on malignancy individually and jointly. We found that when other metabolic traits were present with dyslipidemia, there was no increase in the odds of GBC. In contrast, after excluding the interaction of diabetes and obese, the effects of dyslipidemia on GBC were stronger (adjusted OR 1.915 vs 2.525). Previous studies reported that type 2 diabetes increased the risk of GBC [26]. In our cohort, when interference of obesity was excluded, dyslipidemia was associated with a 2.5-fold increase in the odds of GBC, which was higher than any other metabolic syndrome components. In contrast, obesity was a protective factor for GBC. Dyslipidemia with obesity had no statistical significance $(P=0.631)$. Identifying these metabolic syndrome components in clinical practice should not be too challenging. Thus, these metabolic risk factors we identified, especially dyslipidemia, may serve as important targets for secondary prevention to modify the progression of malignancy in patients with gallbladder lesions.

Low HDL level was associated with an approximately 3-fold increase in the risk of malignancy for patients with gallbladder lesions in this study (Table 5). We also identified that low HDL was associated with a 5-fold increase in the risk of malignancy for patients with neoplastic gallbladder lesions (Supplementary Table 2). HDL is a heterogeneous mixture of macromolecules [27] and has various functions including anti-atherosclerotic, anti-oxidative, anti-inflammatory, anti-thrombotic and anti-apoptotic, as well as involving in immune modulation and endothelial protection [28]. The role of HDL in cancer is still controversial. Some suggest their anti-cancer functions [29]. While other studies reported the association between HDL and the risk of obesity-related cancers, and revealed an association between low HDL level and cancer risk [21]. Decreased HDL was suggested as a hallmark of cancer-induced dyslipidemia [30], which was due to cancer-related inflammation and cholesterol effluxion toward the sites of cancer [31]. It has also been reported that low HDL is an independent risk factor for BGP [32-34]. Patients with BGP had higher TG and lower HDL levels [32]. Another study revealed that high serum lipid levels played an important role in gallstone development and biliary carcinogenesis [12]. The high TG and low HDL levels were closely associated with increased circulating proinflammatory cytokines, including TNF- $\alpha$, IL-1, IL-6 and reactive oxygen species [12]. Dyslipidemia was associated with the formation and development of gallbladder lesions and gallbladder carcinogenesis and our results also support this association.

Our data may inform intensive malignancy surveillance efforts in patients with gallbladder lesions and dyslipidemia. Based on our results, a hierarchical, stepwise and risk-stratification approach may be provided to investigate more targeted surveillance. For example, prioritising patients with gallbladder lesions and low HDL levels for more frequent follow-ups may be more effective and cost effective for early detection of GBC.

This study has some limitations. This is a retrospective analysis of enrolled patients from three 
hospitals in Shanghai. The generalisability of our results needs further validation. Some characteristics including tumor markers, gallbladder wall thickening, and patient lifestyles were not record in our multicenter database and not included in the analysis. The number of patients in GBC group is small, so there might be risks of bias. Although the metabolic syndrome components present targets for secondary prevention of malignancy, our data do not examine treatments. Future clinical trials of risk reduction will be able to reveal if such strategies are effective.

In conclusion, this is the first study indicates that dyslipidemia is associated with an increased risk of malignancy in patients with gallbladder lesions, regardless of the presence of hypertension, diabetes and obesity. Our findings highlight that patients with gallbladder lesions and low HDL levels may need more frequent follow-ups for early detection of GBC, and may be important target for secondary prevention.

\section{Abbreviations}

BGP: Benign gallbladder polyps; GBC: Gallbladder cancer; TG: Triglycerides; HDL: High-density lipoprotein; BMI: body mass index; OR: odds ratio; CI: confidence interval; HDL: high density lipoprotein.

\section{Supplementary Material}

Supplementary tables.

http://www.jcancer.org/v12p1531s1.pdf

\section{Acknowledgements}

This study was funded by the Seeding Grant of Renji Hospital, School of Medicine, Shanghai Jiaotong University (PYZY16-017) and Foundation of Shanghai Municipal Health Commission (202040289).

\section{Ethics Committee Approval}

This study was approved by the Institutional Ethical Review Committee of Renji Hospital, School of Medicine, Shanghai Jiaotong University (Ethical Approval Number 2016-045).

\section{Author Contributions}

Study design: Tao Chen, Haolu Wang, Xiaowen Liang, William J Crawford, Anastasia Brooks; Data collection: Zheng Deng, Yijue Zhang, Zhiqing Yuan, Yan Xuan, Xinxing Li; Statistical analysis: Zheng Deng, Haolu Wang; Statistical process corrected: Yanyan Song; Paper revised: William J Crawford, Anastasia Brooks, Xiaowen Liang. Manuscript: Zheng Deng, Xiaowen Liang.

\section{Competing Interests}

The authors have declared that no competing interest exists.

\section{References}

1. Elmasry M, Lindop D, Dunne DF, Malik H, Poston GJ, Fenwick SW. The risk of malignancy in ultrasound detected gallbladder polyps: A systematic review. International journal of surgery (London, England). 2016; 33(Pt A): 28-35.

2. Stinton LM, Shaffer EA. Epidemiology of gallbladder disease: cholelithiasis and cancer. Gut and liver. 2012; 6: 172-87.

3. Park JY, Hong SP, Kim YJ, Kim HJ, Kim HM, Cho JH, et al. Long-term follow up of gallbladder polyps. Journal of gastroenterology and hepatology. 2009; 24: 219-22.

4. Wiles R, Thoeni RF, Barbu ST, Vashist YK, Rafaelsen SR, Dewhurst C, et al. Management and follow-up of gallbladder polyps: Joint guidelines between the European Society of Gastrointestinal and Abdominal Radiology (ESGAR), European Association for Endoscopic Surgery and other Interventional Techniques (EAES), International Society of Digestive Surgery - European Federation (EFISDS) and European Society of Gastrointestinal Endoscopy (ESGE). European radiology. 2017; 27: 3856-66.

5 Patel $K$, Dajani $K$, Vickramarajah $S$, Huguet $E$. Five year experience of gallbladder polyp surveillance and cost effective analysis against new European consensus guidelines. HPB : the official journal of the International Hepato Pancreato Biliary Association. 2019; 21: 636-42.

6. Wu XS, Shi LB, Li ML, Ding O, Weng H, Wu WG, et al. Evaluation of two inflammation-based prognostic scores in patients with resectable gallbladder carcinoma. Ann Surg Oncol. 2014; 21: 449-57.

7. Li M, Zhang S, Wang Z, Zhang B, Wu X, Weng H, et al. Prognostic significance of nemo-like kinase (NLK) expression in patients with gallbladder cancer. Tumour Biol. 2013; 34: 3995-4000

8. Xu Q, Tao LY, Wu Q, Gao F, Zhang FL, Yuan L, et al. Prevalences of and risk factors for biliary stones and gallbladder polyps in a large Chinese population. HPB : the official journal of the International Hepato Pancreato Biliary Association. 2012; 14: 373-81.

9. Shebl FM, Andreotti G, Rashid A, Gao YT, Yu K, Shen MC, et al. Diabetes in relation to biliary tract cancer and stones: a population-based study in Shanghai, China. British journal of cancer. 2010; 103: 115-9.

10. Yang JI, Lee JK, Ahn DG, Park JK, Lee KH, Lee KT, et al. Predictive Model for Neoplastic Potential of Gallbladder Polyp. Journal of clinical gastroenterology. 2018; 52: 273-6.

11. Chen M, Cao J, Bai Y, Tong C, Lin J, Jindal V, et al. Development and Validation of a Nomogram for Early Detection of Malignant Gallbladder Lesions. Clinical and translational gastroenterology. 2019; 10: e00098.

12. Andreotti G, Chen J, Gao YT, Rashid A, Chang SC, Shen MC, et al. Serum lipid levels and the risk of biliary tract cancers and biliary stones: A population-based study in China. International journal of cancer. 2008; 122 : 2322-9.

13. Andrén-Sandberg A. Diagnosis and management of gallbladder polyps. North American journal of medical sciences. 2012; 4: 203-11.

14. Ito H, Matros E, Brooks DC, Osteen RT, Zinner MJ, Swanson RS, et al. Treatment outcomes associated with surgery for gallbladder cancer: a 20-year experience. Journal of gastrointestinal surgery : official journal of the Society for Surgery of the Alimentary Tract. 2004; 8: 183-90.

15. Wu XS, Wang F, Li HF, Hu YP, Jiang L, Zhang F, et al. LncRNA-PAGBC acts as a microRNA sponge and promotes gallbladder tumorigenesis. EMBO Rep. 2017: 18: 1837-53.

16. Kondo S, Takada T, Miyazaki M, Miyakawa S, Tsukada K, Nagino M, et al. Guidelines for the management of biliary tract and ampullary carcinomas: surgical treatment. Journal of hepato-biliary-pancreatic surgery. 2008; 15: 41-54.

17. Isambert M, Leux C, Metairie S, Paineau J. Incidentally-discovered gallbladder cancer: When, why and which reoperation? Journal of visceral surgery. 2011; 148: e77-84.

18. Rahmani B, Gandhi J, Joshi G, Smith NL, Reid I, Khan SA. The Role of Diabetes Mellitus in Diseases of the Gallbladder and Biliary Tract. Current diabetes reviews. 2020: 16: 931-48.

19. Muszynska C, Nilsson J, Lundgren L, Lindell G, Andersson R, Sandstrom P, et al. A risk score model to predict incidental gallbladder cancer in patients scheduled for cholecystectomy. American journal of surgery. 2020.

20. Muszynska C, Lundgren L, Lindell G, Andersson R, Nilsson J, Sandstrom P, et al. Predictors of incidental gallbladder cancer in patients undergoing cholecystectomy for benign gallbladder disease: Results from a population-based gallstone surgery registry. Surgery. 2017; 162: 256-63.

21. Melvin JC, Holmberg L, Rohrmann S, Loda M, Van Hemelrijck M. Serum lipid profiles and cancer risk in the context of obesity: four meta-analyses. Journal of cancer epidemiology. 2013; 2013: 823849.

22. Singh S, Singh PP, Singh AG, Murad MH, Sanchez W. Statins are associated with a reduced risk of hepatocellular cancer: a systematic review and meta-analysis. Gastroenterology. 2013; 144: 323-32. 
23. Zhang J, Zhang Y, Liu H, Bai H, Wang Y, Jiang C, et al. Antioxidant properties of high-density lipoproteins are impaired in women with polycystic ovary syndrome. Fertility and sterility. 2015; 103: 1346-54.

24. Chahil TJ, Ginsberg HN. Diabetic dyslipidemia. Endocrinology and metabolism clinics of North America. 2006; 35: 491-510, vii-viii.

25. Kang C, LeRoith D, Gallagher EJ. Diabetes, Obesity, and Breast Cancer. Endocrinology. 2018; 159: 3801-12.

26. Gu J, Yan S, Wang B, Shen F, Cao H, Fan J, et al. Type 2 diabetes mellitus and risk of gallbladder cancer: a systematic review and meta-analysis of observational studies. Diabetes/metabolism research and reviews. 2016; 32: 63-72.

27. Jiang JT, $\mathrm{Xu} \mathrm{N}, \mathrm{Wu} \mathrm{CP}$. Metabolism of high density lipoproteins in liver cancer. World journal of gastroenterology. 2007; 13: 3159-63

28. Pirro M, Siepi D, Lupattelli G, Roscini AR, Schillaci G, Gemelli F, et al. Plasma C-reactive protein in subjects with hypo/hyperalphalipoproteinemias. Metabolism: clinical and experimental. 2003; 52: 432-6.

29. Jafri $\mathrm{H}$, Alsheikh-Ali AA, Karas RH. Baseline and on-treatment high-density lipoprotein cholesterol and the risk of cancer in randomized controlled trials of lipid-altering therapy. Journal of the American College of Cardiology. 2010; 55: $2846-54$.

30. Dilman VM, Berstein LM, Ostroumova MN, Tsyrlina YV, Golubev AG. Peculiarities of hyperlipidaemia in tumour patients. British journal of cancer. 1981; 43: 637-43.

31. Kuliszkiewicz-Janus M, Malecki R, Mohamed AS. Lipid changes occuring in the course of hematological cancers. Cellular \& molecular biology letters. 2008; 13: $465-74$

32. Zhao X, Zheng H, Shan S, Wang $K$, Zhang M, Xie S, et al. Association between the non-HDL-cholesterol-to-HDL-cholesterol ratio and the risk of gallbladder polyp formation among men: a retrospective cohort study. Lipids in health and disease. 2020; 19: 146.

33. Yang HL, Kong L, Hou LL, Shen HF, Wang Y, Gu XG, et al. Analysis of risk factors for polypoid lesions of gallbladder among health examinees. World journal of gastroenterology. 2012; 18: 3015-9.

34. Yamin Z, Xuesong B, Zhen Z, Yue H, Liwei L, Fei L. Correlation of dyslipidemias and gallbladder polyps-A large retrospective study among Chinese population. Asian journal of surgery. 2020; 43: 181-5. 